\title{
AÇÕES DE SAÚDE MENTAL NA REGIÃO NORTE DO RIO GRANDE DO SUL, BRASIL
}

Ricardo Vianna M ART IN Sa, M aíra ROSSET T O ${ }^{b}$, Queli D aiane N ogueira SART ORIc, É der Campos PIN T O ${ }^{d}$ I sabel Cristina Pacheco VAN DE'R SAN De, Leila M ariza HILDEBRANDT ${ }^{f}$

\section{RESUMO}

O objetivo deste estudo é descrever as ações e projetos de intervenção em saúde mental nos serviços de saúde existentes nos municípios da área de abrangência da 15a Coordenadoria Regional de Saúde. T rata-se de pesquisa qual itativa, exploratória, descritiva. Os dados foram col etados por meio de questionário, com profissionais e gestores que participam de fóruns regionais de saúde mental. A partir da análise do conteúdo emergiram categorias: uma aborda a descrição da atenção em saúde mental nos serviços de saúde, incluindo aspectos relativos aos transtornos mentais, a organização da equipe de saúde mental, as ações desenvolvidas e às dificuldades encontradas; a outra trata dos projetos de interven ção em saúde mental que, de acordo com os sujeitos, deverão ser implementados nos municípios. Foram identificados pontos relevantes da rede regional de atenção na saúde mental que poderão auxiliar no planejamento e na melhoria da assistência.

D escritores: Saúde mental. Serviços de saúde. Pessoal de saúde.

\section{RESUMEN}

E I objetivo de este estudio es describir las actividades y proyectos de inter vención en salud mental en los servicios de salud en los municipios de la zona cubierta por la 15a R egional de Salud. Setrata de un estudio cualitativo, exploratorio y descriptivo. L os datos fueron recolectados en un cuestionario, con profesionales que participan en los foros regionales sobre la salud mental. A través del análisis de contenido se llegó a dos categorías: una trata de la descripción de la atención de la salud mental en los servicios, induidas las cuestiones relacionadas con los trastornos mentales, la organización del personal de salud mental, las medidas adoptadas y las dificultades encontradas; otro trata de proyectos de inter vención en salud mental que, deacuerdo con los sujetos, deben aplicarse en los municipios. Seidentificar on los puntos relevantes dela red regional deatención en salud mental.

Descriptores: Salud mental. Servicios de salud. Personal de salud.

T ítulo: A cciones de la salud mental en la región nor te de R io G rande do Sul, B rasil.

\section{ABST RACT}

The objective this study is to describe the actions and intervention projects in mental health in the health ser vices of the cities that are part of R io G randedo Sul's 15th R egional H ealth Coordinating B ody (15a Coordenadoria R egional deSaúde). T his is an explorator $y$, descriptive and qualitative research. A questionnaire was applied to health professi onals and managers who were part of regional mental health for ums. F rom the content anal ysis as proposed by $M$ inayo, thefollowing categories emerged: one which describes mental health carein thehealth services of themunici palities, induding aspects related to mental disorders, to the organization of the mental health team, to the main developed actions and to the difficulties found; the other category is about intervention projects in mental health that, according to the subjects, will be implanted in the cities. Re elevant points the regional netw ork of mental health care w ere identified, which will be helpful in planning and improving care.

D escriptors: $M$ ental health. $\mathrm{H}$ ealth services. $\mathrm{H}$ ealth per sonnel.

T itle: $\mathrm{M}$ ental health actions in the north of the state of $\mathrm{R}$ io $\mathrm{G}$ rande do $\mathrm{Sul}, \mathrm{B}$ razil.

a D outor em Psicologia, D ocente do Curso de Enfer magem do Centro de E ducação Superior do N orte do Rio G rande do Sul (CESN ORS) da U niversidade Federal de Santa M aria (U F SM ), Palmeira das M issões, Rio G rande do Sul, Brasil.

b Enfermeira graduada pelo CE SN ORS/ U F SM, Porto Alegre, Rio Grande do Sul, Brasil.

'E nfer meira graduada pelo CESN ORS/ U FSM, Palmeira das M issões, Rio G rande do Sul, Brasil.

¿Enfer meiro graduado pelo CESN ORS/ U F SM, Porto Alegre, Rio Grande do Sul, Brasil.

e M estre em Enfermagem, D ocente do Curso de Enfermagem do CESN ORS/ UFSM, Palmeira das M issões, Rio Grande do Sul, Brasil.

f M estre em Enfermagem Psiquiátrica, D ocente do Curso de Enfermagem do CESN ORS/ U FSM, Palmeira das M issões, Rio Grande do

Sul, Brasil. 


\section{INT RODUÇÃO}

A saúde mental vem enfrentando mudança paradigmática desde meados do século passado, momento em que os portadores de sofrimento psiquiátrico eram segregados da sociedade, enclausurados em manicômios, com tratamento baseado em preconceitos científicos e violações aos direitos humanos ${ }^{(1)}$.

Esse modelo assistencial, excludente e segregador, perpetuou-se por longos anos. Somente a partir da década de 1970, desencadearam-se al guns movimentos no Brasil apontando críticas ao modelo manicomial no que se refere à assistência psiquiátrica ${ }^{(2)}$. $\mathrm{Na}$ década de $80,0 \mathrm{M}$ ovimento pela $\mathrm{Re}$ forma Psiquiátrica Brasileira ganhou mais força, pois se uniram à luta diferentes atores sociais, como usuários dos serviços, família, profissionais da saúde e sociedade em geral, os quais passaram a integrar as discussões e consolidar a L uta Antimanicomial. A partir daí, ocorreram várias mudanças nas políticas, na legislação e na organização da atenção em saúde mental, apontando a necessidade de equipamentos múltiplos para atender à complexidade do sofrimento psíquico(3).

Esse novo modelo de atenção é pautado na valorização do ser humano e na desinstitucionalização do portador de transtorno mental, incluindo-o na sociedade e oportunizando novas formas de cuidado que superem a intensa medicalização/ alienação do sujeito.

Neste contexto, a saúde mental brasileira passa, há mais de uma década, por adequações em seu modelo assistencial e estrutura suas redes para prestar cuidado qualificado em todas as suas instâncias: tanto ambulatorial quanto hospitalar. Nessa proposição, é fundamental o trabalho em rede com uma intensa comunicação entre os serviços, os profissionais e os usuários/ familiares visando à qualificação da atenção.

A atenção em saúde mental no Brasil, de acordo com as prerrogativas do M inistério da Saúde, deve se estabelecer em consonância com os princípios e as diretrizes do Sistema Ú nico de Saúde (SU S), envolvendo a rede de atenção básica, os serviços substitutivos e os hospitais gerais. $M$ ais do que uma política de resultados ou de "cura", importa que os sujeitos que chegam a esses serviços tenham, antes de tudo, um acolhimento nas suas singularidades e encontrem aí uma referência(4).
0 Centro de Atenção Psicossocial (CAPS) constitui-se num dos serviços substitutivos, com um atendimento diário em que o usuário segue projeto terapêutico estabelecido, em comum acordo, entre el e, sua família e a equipe do serviço. I sso implica a manutenção da convivência do usuário com a família e a sociedade. Os CAPSs, criados oficial mente pela Portaria 336/ $2002^{(5)}$, têm como meta dar andamento objetivo às propostas oriundas da reforma psiquiátrica, que repudiam o modelo asilar excludente e segregador.

Outros serviços também podem ser mencionados, tais como as residências ter apêuticas e o programa de volta para casa, que incentivam os portadores de transtornos mentais a terem independência e vida própria, recebendo apoio dos profissionais da saúde e da comunidade. Assim, tanto na atenção básica com os serviços substitutivos quanto na inter nação hospitalar, não se admite institucionalização do portador de transtorno mental.

Para além desses serviços, neste novo modeIo assistencial é esper ado que a rede de atenção básica, incluindo a Estratégia de Saúde da Família, acompanhe as pessoas portadoras de sofrimento mental e sua rede relacional, oportunizando-Ihes 0 suporte de que necessitam.

Pela portaria no 1174, de 7 de julho de $2005^{(6)}$ há destinação de recursos para que os municípios desenvolvam o P rograma de Q ual ificação do A tendimento e da G estão dos CAPS. N este contexto, a portaria prevê o desenvolvimento de pesquisas e a produção de conhecimento articulados com instituições formadoras de recursos humanos. Assim, baseados principal mente nessa recomendação e na demanda da 15a Coordenadoria Regional de Saúde (CRS-RS), que solicitava que a univer sidade colabor asse na implantação e organização dos ser viços municipais de atendimento à saúde mental, pensou-se na estruturação de uma rede entre os profissionais da área de saúde mental - psicólogos, psiquiatras, enfer meiras, assistentes sociais, técnicos, etc. - com vistas à troca de informações e à constituição de um grupo com o propósito de discutir e fomentar a formação continuada em saúde mental, mediante cursos, capacitação técnica e humanística, seminários, grupos de estudos e pesquisa.

N essa direção, esta pesquisa, al iada a um projeto de extensão, teve como objetivo descrever as ações e projetos de intervenção em saúde mental nos serviços de saúde existentes nos municípios 
da área de abrangência da 15a CRS. 0 estudo poderá contribuir para a formulação de propostas de trabal ho no campo da saúde mental e para o fortalecimento das já existentes nos municípios adstritos à 15a CRS.

\section{METODOLOGIA}

E ste estudo se caracteriza como uma pesquisa qualitativa, descritiva e exploratória ${ }^{(7,8)}$. Para a coleta dos dados, aplicou-se um questionário com seis questões abertas, que foram respondidas pelos integ rantes das equipes multiprofissionais de saúde e gestores dos municípios de abrangência da 15a CRS. 0 foco incidiu sobre a real idade da saúde mental de cada município, as ações desenvolvidas nessa área, a formação e composição das equipes de saúde, as dificuldades encontradas e os projetos de intervenção planejados para a área de cada município.

D e um total de 26 municípios, 23 devolveram o questionário respondido. Os sujeitos deste estudo foram os 70 profissionais de diferentes categorias dos municípios respondentes, dentre os quais 18 enfermeiros, 11 agentes comunitários de saúde, 10 técnicos e auxiliares de enfer magem, oito odontólogos, sete assistentes sociais, seis psicólogos, cinco médicos, três fisioterapeutas e dois terapeutas ocupacionais, e os 23 gestores de saúde desses municípios. Os questionários foram aplicados durante o II Fórum de Saúde M ental, promovido em parceria entre a 15 CR S e a U niversidade F ederal de Santa M aria, campus do Centro de Educação Superior do Norte do R io G rande do Sul (UF SM / CESNORS).

As informações coletadas por meio do questionário foram ordenadas, classificadas e analisadas segundo a proposta da análise do conteúdo ${ }^{(7)}$, que prevê as seguintes etapas: pré-análise, exploração do material, tratamento dos resultados obtidos e inter pretação. Por meio do processo analítico, os dados foram agrupados em categorias, em que a primeira trata da descrição dos serviços de saúde mental dos municípios da área de abrangência da 15a Coordenadoria Regional de Saúde e a segunda aborda os projetos de intervenção em saúde mental dos referidos serviços.

A pesquisa seguiu os preceitos éticos estabelecidos pela Resolução 196/ 96 do Conselho N acional de Saúde ${ }^{(9)}$ e foi aprovada pelo Comitê de É tica e Pesquisa da U niversidade Federal de Santa M a- ria, sob protocolo no 0095.0.243.000-10. Os indivíduos participantes assinaram o Termo de Consentimento Livre e Esclarecido (T CLE ), que foi arquivado juntamente com os questionários, que ficarão à disposição de todos os interessados pelo período de cinco anos.

\section{RESULT ADOS E DISCUSSÃO}

Buscando atender ao objetivo desta pesquisa, através das informações advindas do estudo emergiram categorias, uma descreve as ações em saúde mental nos serviços de saúde e a outra aborda os projetos de inter venção em saúde mental.

\section{A saúde mental nos serviços de saúde dos municípios da área de abrangência da 15ạ Coordenadoria Regional de Saúde}

As ações de atenção à saúde mental são orientadas, em parte, pelos tipos de transtor nos mentais mais frequentes em cada real idade. A ssim, buscou-se identificar tais transtornos, constatando-se que os relacionados ao uso de substâncias psicoativas mostraram-se mais frequentes, a exemplo de álcool e drogas (crack e cigarro). T ambém foram referenciados a esquizofrenia, o transtor no afetivo bipolar e a depressão.

0 consumo de ál cool e outras drogas está inserido no cotidiano de grande parte da população mundial. Tal realidade está associada a uma série de situações de risco à saúde e vem sendo observada em diferentes países. Indicativos mostram que cerca de $10 \%$ da população dos centros urbanos de todo o mundo consome abusivamente substâncias psicoativas, independentemente de idade, sexo, nível de instrução e poder aquisitivo. Salvo variações sem repercussão epidemiológica significativa, essa real idade encontra equivalência em ter ritório brasileiro(10)

Q uanto à organização da equipe de saúde mental e à forma como esta atua, os profissionais dos municípios relatam possuir equipes, na sua maioria compostas por enfermeiros, psicólogos, médicos e assistentes sociais, ou organizadas conforme a lógica da Estratégia de Saúde da Família. Algumas contam, ainda, com fisioterapeuta, agentes comunitários de saúde, técnicos de enfermagem, extensionistas rurais, visitadoras da Primeira Infância M elhor, Grupo de Alcoólicos Anônimos, consel heiro tutelar, entre outros. 
Dos dados, emerge que o trabalho das equipes está pouco articulado, na medida em que cada profissional trabal ha na sua área de atuação e não ocorre planejamento ter apêutico para a população atendida. Assim, existe um coletivo que formal mente se estrutura em equipes, mas, de maneira geral, se individualiza e pulveriza sua eficiência, enfrentando dificuldades em atuar no planejamento e organização de ações em saúde mental.

Os profissionais das equipes relatam que:

Sim, possuímos equipe, composta por enfermeira, mé dico, técnico de enfermagem, psicóloga, assistente social, fisioterapeuta, atuando como E SF (G21).

$\mathrm{N}$ ão há uma equipe formada, por isso as dificuldades se tornam relevantes [ ...] (G 15).

A proposta do trabalho em equipe tem sido veiculada como estratégia para enfrentar o intenso processo de especialização na ár ea da saúde, que tende a apr ofundar vertical mente o conhecimento e a intervenção em aspectos individualizados das necessidades de saúde, sem contemplar simultaneamente a articulação das ações e dos saberes ${ }^{(11)}$. Para que tal verticalização do conhecimento não aconteça, é importante que os profissionais trabalhem com uma lógica de equipe, planejem e implementem medidas de cuidado, valorizando cada área de conhecimento. D esse modo, há necessidade de encontro físico e conceitual, sistemático, entre os diferentes profissionais, com vistas à assistência qual ificada, pautada por um referencial comum entre a equipe, o usuário e sua família.

Para trabalhar nessa lógica de equipe, observa-se a relevância do desenvolvimento de Projeto Terapêutico Singular (PT S), que engloba a participação de todos os profissionais, do usuário e sua família, no intuito de fortalecer o vínculo e a confiança entre os atores sociais envolvidos nessa relação. O PT S consiste em encontro em que todas as opiniões são importantes para ajudar a entender 0 sujeito doente e sua relação com a doença, para a definição de propostas de ações ${ }^{(12)}$. Essa forma de inter venção é utilizada, na atenção em saúde mental, como meio de propiciar atuação integrada da equipe de saúde e de super ar a visão vertical da doença, na medida em que trabal ha com o conceito de clínica ampliada e visa o atendimento do usuário em sua integralidade.

No tocante às ações desenvolvidas no campo da saúde mental nos diferentes municípios parti- cipantes do estudo, os resultados apontam para as visitas domiciliárias, grupos de apoio e, ainda, 0 atendimento individual e familiar. A visita domiciliária se constitui numa atividade adotada com 0 intuito de subsidiar a intervenção no processo de saúde-doença de indivíduos ou no planejamento de ações visando à promoção da saúde da coletivida$\mathrm{de}^{(13)}$.

Esse instrumento é um facilitador da abordagem dos usuários e sua família. Por meio desse recurso, pode-se entender a dinâmica familiar, com 0 objetivo de verificar as possibilidades de envolvimento da família no tratamento oferecido ao usuário. E mais, pode fornecer aos usuários dos serviços de saúde mental suporte para que possam dar continuidade ao tratamento, evitando assim a reinternação(14).

Os grupos de apoio, em sua maioria, buscam o acompanhamento do paciente e de sua família e, ao mesmo tempo em que se constituem prática terapêutica, também proporcionam a criação de vínculos afetivos entre participantes e profissionais da saúde, o que auxilia no processo de tratamento. 0 trabalho com grupos constitui um recurso terapêutico importante no campo de saúde mental. E sse dispositivo terapêutico passou a ser mais utilizado a partir da reforma psiquiátrica, tendo por foco a ressocialização do indivíduo em sofrimento psíquico(15).

Os dados também permitem inferir que 0 atendimento individual e familiar é realizado, na maioria das vezes, pelo psicólogo da unidade básica de saúde de referência. No contexto do estudo, consiste em acompanhamento mensal ou quando o usuário deflagra uma crise. Nos municípios estudados não há psiquiatras vinculados às unidades básicas de saúde, havendo atendimento por médicos clínicos gerais, o que vai ao encontro dos pressupostos da reforma psiquiátrica e da atenção básica em saúde. N esse espaço, o médico clínico geral tem condições de ofertar o suporte demandado pelos usuários portadores de doença mental, já que, na crise, estes são encaminhados para serviços especializados.

Quanto às dificuldades enfrentadas pel os profissionais na resolução dos problemas de saúde mental, os dados indicam a resistência da família em relação ao tratamento da pessoa portadora de transtorno mental, quando muitas não permitem que o familiar doente seja assistido ou dificultam 0 seu acesso ao tratamento. Ainda citam a falta de 
planejamento e organização da rede de referência e contrar referência dos municípios.

R eferente à participação da família, os profissionais relatam que:

Falta comprometimento familiar eque el es assumam 0 paciente [...] (G3).

P recisamos de mais responsabilidade dos familiares junto ao tratamento [ ...] (G11).

D epois da reforma psiquiátrica, a saúde mental é pautada no modelo psicossocial, que, dentre outras características, considera o usuário do serviço como uma pessoa em sofrimento psíquico, cujo tratamento deve se dar com seus familiares e no meio social em que vive. Neste sentido, a família deverá exercer um papel de protagonista do cuidado reabilitador psicossocial, sendo parceira no trabal ho de atenção à saúde mental(16). D este modo, a família, assim como usuário portador de transtor no mental, também precisa de acompanhamento e suporte para realizar o cuidado.

A família é vista pelos profissionais da saúde como uma aliada nos cuidados e destaca-se a dificuldade em trabal har sem seu apoio. No campo da reforma psiquiátrica, a família é considerada um valioso instrumento para a assistência de base comunitária ${ }^{(17)}$. A ssim, um projeto de assistência deve ser pensado pela família e equipe de saúde e ambas devem participar do processo de cuidado do usuário. Ressalta-se, ainda, que a família também deverá ser considerada um núcleo a ser cuidado.

Referente às redes de assistência, os sujeitos do estudo mencionam que elas precisam ser meIhoradas:

Falta planejamento da rede [...] (G 4).

Temos fal has no pós-alta, édifícil o acompanhamento [...] (G5).

E ncaminhamos o paciente para uma internação, mas quando ele volta não sabemos o que aconteceu na internação, os medicamentos que usou, os cuidados necessários [ ...] (G 13).

A rede pode ser entendida como um conjunto de nós inter conectados ${ }^{(18)}$, que ganha potência se a principal referência para sua produção for o território, pois é nel e que se dá a articul ação entre diferentes serviços, "os nós da rede" para a formação das diversas redes de assistência. A rede de atenção frag mentada tem, como consequência, 0 "abandono" da comunidade, pois ao selecionar os problemas com base em uma especialidade, ou, pior, segundo um tipo de "doença mental", as pessoas são abandonadas a si mesmas, com suas vivências, muitas vezes, problematizadas. 0 vínculo terapêutico, quando existe, é frágil e a responsabilização não é clara.

Seguindo essa lógica de construção de redes segundo um território, trabalha-se com um princípio do Sistema Ú nico de Saúde (SU S), a regionalização da atenção à saúde, que cria espaços assistenciais de uso comum por diver sos atores. $\mathrm{N}$ a região norte do estado, ter ritório deste estudo, podese afirmar que há uma ruptura na conectividade dos nós da rede, pois as ações são fragmentadas e os profissionais dos diferentes pontos da rede se inter-relacionam minimamente, fato importante que necessita ser superado.

\section{Projetos de intervenção em saúde mental}

Este tema descreve, a partir dos dados obtidos junto aos sujeitos do estudo, as ações que os municípios pretendem desenvolver para a estruturação da atenção em saúde mental. A adoção, neste estudo, do termo "projetos de intervenção" faz referência as ações de cuidado que o ser viço de saúde mental de cada município planeja implementar, dentro de uma rede de atenção à saúde regional que se entrelaça, para o assistência aos usuários em sofrimento psíquico. Assim, faz-se a descrição de projetos de intervenção em saúde mental dos municípios e da rede regional de saúde mental.

Quanto aos projetos de inter venção em saúde mental que estão sendo pensados para os municípios da área de abrangência da 15 a CRS, os respondentes indicam que a formação e ampliação da equipe em saúde mental, bem como a composição de grupos terapêuticos de tabagistas, al coolistas e de autoajuda estão em destaque. Ainda, são citados os projetos de criação de Centros de A tenção Psicossocial (CAPS) em dois municípios com mais de 20.000 habitantes, conforme previsto na portaria $336 / 2002^{(5)}$, ou seja, CA PS I. Outra pretensão é a organização de acompanhamento após a alta hospitalar dos indivíduos portadores de transtorno mental, com a possibilidade de inserção comunitá- 
ria e no campo de trabal ho, com vistas à prevenção de agravos em saúde mental.

Referente aos projetos de intervenção a serem implementados, os sujeitos do estudo assim se posicionam:

F ormar uma equipe de saúde mental, com todos sendo capacitados para este fim, melhorando assi $m 0$ atendimento ao usuário (G 15).

D esejamos criar um centro de atendimento em saúde mental em nível local (G 4).

E stamos em andamento com o grupo deal coolismo, que está dando cer to, reativação do grupo de AA, grupo de tabagismo, bem como estamos encaminhando o grupo de pacientes psiquiátricos (G 12).

Grupo de apoio para dependente químico e seus familiares, bem como para os reingressos, re internaçōes psiquiátricas, palestras educativas visando à prevenção no uso de substâncias químicas (G21).

Visitas residenciais, qualificação da equipe, grupos terapêticos, envolvimento da família no tratamento (G 22).

Como observado, cada município da região da 15a CRS, representado neste estudo, constrói seu projeto de atenção em saúde mental baseado em suas necessidades e demandas imediatas. $M$ as um projeto mais amplo é pensado para a região, como, por exemplo, a construção de uma rede de assistência que atenda aos usuários e familiares, bem como, a implantação dos CA PSs. Essa rede de assistência deve possuir um fluxograma dos usuários, ou seja, por onde esses indivíduos precisam transitar em busca de tratamento, tanto na rede ambulatorial quanto hospitalar.

O utro desafio importanteé implementar equipes de saúde mental nas unidades básica de saúde e unidades de saúde da família, qualificadas para atender esse tipo de demanda. A lém disso, há a necessidade de aumentar o número de leitos em hospitais gerais para internação das pessoas quando da agudização dos sintomas da enfermidade mental, observando protocolos de entrada e saída em cada serviço de atenção, para que o usuário e o tratamento não se percam "no meio do caminho". T odo esse fluxo deverá ser organizado pela 15a CRS.

Cabe salientar que os profissionais de saúde mental, os gestores, os profissionais vinculados à 15a CRS e docentes e discentes da U niversidade inserida no contexto do estudo estão articulados no intuito de planejar e organizar a assistência em saúde mental, vislumbrando as propostas do M inistério da Saúde relativas à desinstitucional ização e apoio à estruturação de serviços substitutivos. $A$ organização da rede de assistência é apontada, pelos sujeitos estudados, como uma possível medida para a atenção qualificada aos usuários e seus familiares.

No contexto dos cuidados em saúde mental, os serviços substitutivos se revelam como propostas inovadoras, transformadoras e alternativas viáveis para redefinir a atenção psiquiátrica hospital ocêntrica. Constituem, ainda, uma proposta que, al ém de inovadora, repolitiza o sujeito e a saúde, de modo a produzir movimentos contra ideológicos e contra-hegemônicos no macro espaço - o contexto das políticas públicas - e no micro espaço - quando al cança os processos de trabal ho das equipes, a organização dos serviços, a redefinição das práticas dos trabalhadores e as relações que estabelecem com os sujeitos que os procuram ${ }^{(19)}$.

As ações com vistas à ampliação da rede de assistência, juntamente com um atendimento qualificado e condizente com as propostas do M inistério da Saúde, favorecem a integ ralidade da atenção e valorizam os usuários acometidos por transtornos psiquiátricos e seus familiares, com incentivo a sua autonomia e val orização da vida. Outras atividades incluem a promoção da saúde por meio de ações de educação em saúde e prevenção de usos de substâncias psicoativas entre a população, especial mente crianças e adolescentes.

\section{CONCLUSÕES}

Posto que o objetivo deste estudo foi descrever as ações e projetos de intervenção em saúde mental nos serviços de saúde existentes nos municípios da área de abrangência da 15a Coordenadoria Regional de Saúde, identificou-se, quanto aos serviços, que as equipes de saúde se encontram pouco estruturadas e realizando ações fragmentadas com atividades como as visitas domiciliárias, grupos de apoio e 0 atendimento individual e familiar. Encontram também dificul dades relacionadas à resistência familiar no tratamento do portador de transtor no mental e à falta de rede de assistência organizada.

Ainda, quanto aos projetos de intervenção em saúde mental, al gumas ações são pensadas para 
a melhoria da assistência, tais como a criação de CAPS em municípios que comportam esse serviço, estruturação das equipes de saúde e organização do fluxo de pacientes entre os municípios da região, com a perspectiva da formação de uma rede regulada pela 15a CRS.

Com esta pesquisa, vários dados foram levantados quanto às equipes de saúde mental vinculadas a municípios da abrangência da 15a CRS, suas dinâmicas de trabalho, dificuldades e potencialidades. Os resultados do presente estudo poderão subsidiar o planejamento de atividades nos fóruns regionais de saúde mental a partir das necessidades que emergem do cotidiano de trabal ho destes profissionais, aqui expressas, na perspectiva de contribuir com o fortalecimento da referida área na região.

D estacam-se, neste estudo, alguns fatores limitantes relativos à atenção em saúde mental, dentre os quais a falta de equipes de saúde mental e a ausência de redes assistenciais com referências e contrar referências organizadas e que se comuniquem entre si, bem como a ausência de serviços substitutivos e desinstitucional izantes.

Este trabalho não se encerra em si e deixa margem para novas pesquisas, tendo em vista 0 momento histórico pelo qual passa a saúde mental e o seu amplo contexto na realidade pesquisada. Ressal ta-se, também, a val oração que representa o trabal ho realizado pel os profissionais e gestores em saúde e sua importância para a construção de um sistema de saúde pautado pelos princípios do SU S e os pressupostos da Reforma Psiquiátrica.

\section{REFERÊNCIAS}

1 A breu DN. A prática entre vários: a psicanálise na instituição de saúde mental. Estud Pesqui Psicol. 2008;8(1):74-82.

2 Antos NS, A Imeida PF, Venancio AT, D elgado PG. A autonomia do sujeito psicótico no contexto da reforma psiquiátrica brasileira. Psicol Ciênc Prof. 2000; 20(4):46-53.

3 W etzel C, K antorski LP, Sousa J. Centro de A tenção Psicossocial: trajetória, organização e funcionamento. Rev Enferm UERJ. 2008;16(1):39-45.

4 Ribeiro AM . U ma reflexão psicanalítica acerca dos CAPS: al guns aspectos éticos, técnicos e políticos. Psicol U SP. 2005;16(4):33-56.
5 M inistério da Saúde (BR ). L egislação em saúde mental. 2ª ed. Brasília (DF); 2004.

6 M inistério da Saúde (BR). Portaria no 1174/ G M , de 7 de julho de 2005: destina incentivo financeiro emergencial para o Programa de Q ualificação dos Centros de Atenção P sicossocial-CAPS e dá outras providências. Brasília (DF); 2005.

7 M inayo M CS. 0 desafio do conhecimento: pesquisa qual itativa em saúde. 10ª ed. São Paulo: H ucitec; 2007.

8 T obar F, Yalour M R. Como fazer teses em saúde pública: consel hos e idéias par a for mular projetos e redigir teses e informes de pesquisas. Rio de Janeiro: Fiocruz; 2002.

9 M inistério da Saúde (BR ), Consel ho N acional de Saúde. Resolução 196, de 10 de outubro de 1996: diretrizes enor mas regulamentadoras de pesquisa envolvendo seres humanos. Brasília (DF); 1996.

10 M inistério da Saúde (BR ), Secretaria E xecutiva, Secretaria de A tenção à Saúde. A política do M inistério da Saúde para atenção integral a usuários deálcool e outras drogas. Brasília (D F); 2003.

11 Peduzzi M . Equipe multiprofissional de saúde: conceito e tipologia. Rev Saúde Pública. 2001;35(1):1039.

12 Carvalho SR, Cunha GR. A gestão da atenção na saúde: el ementos para se pensar a mudança da organização na saúde. In: Campos GW S, organizador. T ratado de saúde coletiva. São Paulo: H ucitec; Rio de Janeiro: F iocruz; 2006. p. 837-68.

13 T akahashi RF, Oliveira M AC. A visita domiciliária no contexto da saúde da família. I n: I nstituto para o D esenvolvimento da Saúde; U niversidade de São Paulo; M inistério da Saúde (BR). M anual de enfermagem. Brasília (DF); 2001. p. 43-6.

14 Reinaldo M AS, Rocha RM . Visita domiciliar de enfermagem em Saúde M ental: idéias para hoje e amanhã. Rev Eletrônica E nferm. 2002;4(2):36-41.

15 Sousa A M A, Fraga M N O, M oraes L M P, G arcia M LP, M oura KD R, Almeira PC, et al. G rupo terapêutico: sistematização da assistência de enfer magem em saúde mental. T exto Contexto E nferm. 2004;13(4):62532.

16 Colvero LA, I de CAC, Rolim M A. Família e doença mental: a difícil convivência com a diferença. Rev E sc Enferm USP. 2004;38(2):197-205. 
17 Ribeiro CC, Ribeiro LA, Oliveira AG B. A construção da assistência à saúde mental em duas unidades de saúde da família de Cuiabá-M T. Cogitare E nferm. 2008;13(4):548-57.

18 Righi LB. Poder local e inovação no SU S: estudo sobre a construção de redes de atenção à saúde em três municípios no E stado do R io G rande do Sul [ tese] Campinas: $F$ aculdade de Ciências M édicas, U niversidade de Campinas; 2002.

19 Pinho LB, Her nández A M B, K antorski LP. Serviços substitutivos de saúde mental e inclusão no ter ritório: contradições e potencialidades. Ciênc Cuid Saúde. 2010;9(1):28-35.
E ndereço da autora / Dirección del autor / Author's address:

M aíra Rossetto

Rua Jeronimo de Ornelas, 527, ap. 03, Santana

90040-341, Porto Alegre, RS

E-mail: maira_rossetto@hotmail.com
Recebido em: 16/ 05/ 2011

A provado em: 02/ 03/ 2012 